\title{
STUDY ON RELATIONSHIP BETWEEN STROKE AND KIDNEY DYSFUNCTION BASED ON ESTIMATED GLOMERULAR FILTRATION RATE (eGFR)
}

\author{
SHARMIN JAHAN ${ }^{1}$, ROKEYA BEGUM ${ }^{2}$, QAZI SHAMIMA AKHTER ${ }^{3}$ \\ ${ }^{1}$ Assistant Professor, Department of Physiology, Shaheed Monsur Ali Medical College, Uttara, Dhaka. \\ ${ }^{2}$ Professor and Head of the Department of Physiology, Enam Medical College, Savar, Dhaka. \\ ${ }^{3}$ Professor and Head of the Department of Physiology, Dhaka Medical College, Dhaka.
}

\begin{abstract}
Background: Stroke is one of the commonest causes of severe disability and accounts for a large proportion of health care resources. Kidney diseases are related to stroke and reduced estimated glomerular filtration rate (eGFR) appears to be a significant independent prognostic factor for short as well as long term mortality in stroke patients. Objective: The present study has been designed to assess the relationship between stroke and kidney dysfunction based on eGFR. Methods: The present study was a case control study. The study was conducted in the Department of Physiology, Dhaka Medical College and Hospital (DMCH), Dhaka from 1st July 2010 to 30th June 2011. A total of 200 subjects were included with the age limit of 35-85 years. Out of them 100 apparently healthy subjects were selected as control(Group A) for comparison and 100 diagnosed stroke patients were selected as study group (Group B). On the basis of age, group A and group B were further subdivided into group $A_{1}$ (age 35-59 years) consisting of 47 normal persons and group $A_{2}$ (age 60-85 years) consisting of 53 normal person, group $B_{1}$ (age35-59 years) was consisting of 42 stroke patients and group $B_{2}(60-85$ years) consisting of 58 stroke patients. The study subjects were selected from admitted patients in Department of Medicine, DMCH, Dhaka. Estimated GFR (eGFR) were measured by MDRD equation. The data was analyzed by computer based statistical software (SPSS version 12). Results: The result was expressed as Mean ( $\pm S D)$. The test of significance was calculated and $p$ values $<0.05$ was accepted as level of significance. The mean $( \pm S D)$ of eGFR level was significantly $(p<0.001)$ lower in group $B_{1}$ and $B_{2}$ than that of group $A_{1}$ and $A_{2}$ respectively. Conclusion: From this study it can be concluded that, stroke patients suffers from kidney dysfunction than that of normal control group on the basis of measuring eGFR.
\end{abstract}

Key words: eGFR, stroke, kidney dysfunction.

(Bangladesh J Physiol Pharmacol 2013;29(1\&2):12-16.)

\section{INTRODUCTION}

Stroke is an important health issue for individual and society. According to World Health Organization(WHO), stroke is a clinical syndrome occurring due to sudden cerebral dysfunction producing focal neurological deficit persisting more than 24 hours or the patient die within 24 hours, which is vascular in origin, nonepileptic and non traumatic in nature. In the developed countries, after heart disease and cancer, stroke is the third leading cause of death and it causes serious long term disability. Each year about 4.4 million people die of stroke globally, of whom three millions are from developing countries.

The major risk factors for stroke are hypertension, smoking, diabetes mellitus, hyperlipedemia etc. The other risk factors are high alcohol intake, positive family

Address for correspondence: Sharmin Jahan, Assistant Professor, Department of Physiology, Shaheed Monsur Ali Medical College, Uttara, Dhaka. Email: sjlubna@yahoo.com history, oral contraceptives etc. Recent evidence suggest that, cerebral small vessel disease is closely associated with kidney function in patients with acute stroke and reduced kidney function may predict poor post stroke survival. ${ }^{2}$

Kidney dysfunction is characterized by glomerular endothelial abnormality and lipohyalinosis; both of which are features of small vessel disease of the kidney. White matter lesions (WML), lacunar infracts and subcortical atrophy are markers of cerebral small vessel disease that increases the risk of stroke, cognitive decline and dementia. ${ }^{3}$ Because of the hemodynamic similarities between the vascular beds of the kidney and the brain, small vessel disease in the kidney may be indicative of presence of small vessel disease in the brain. ${ }^{4}$ This relationship is also due to the fact that the blood vessels of both the kidney and the brain have low resistance and are therefore highly susceptible to fluctuations in blood pressure and flow. 
Kidney disease is an important independent risk factor for cerebrovascular disease, Even mild level of kidney dysfunction is associated with white mater abnormalities, Early recognition of chronic kidney disease and prevention of it's progression might prevent the development of white mater abnormalities. ${ }^{5}$

Patients with silent brain infarction (SBI)should be considered a high risk population for decreased kidney function. ${ }^{6}$

Glomerular filtration rate is an important clinical indicator of kidney function. The GFR assess the excretory function of the kidneys and is considered the gold standard to evaluate the renal function.

Measurement of endogenous creatinine clearance to get the GFR requires the concentration of creatinine in serum and urine 24 hours urine volume, body weight. All of these takes time and can be affected by muscle mass. Because of these clinical difficulties estimated GFR (eGFR) is often used in place of creatinine clearance test.

An eGFR , $<60 \mathrm{ml} / \mathrm{min} / 1.73 \mathrm{~m}^{2}$ body surface area represents loss of half or more the adult level of normal kidney function and is associated with an increased complication .7

Low eGFR is an strong independent predictor of mortality and poor outcome in patient with acute stroke. ${ }^{8}$ Among patients with acute ischemic stroke a reduced or highly elevated eGFR at hospital admission is associated a higher mortality rate compared to patients with moderate levels of eGFR. ${ }^{9}$

Reduced estimated GFR appears to be a significant independent risk factor for short as well as long term mortality. So eGFR should be added to the other known prognostic factors and it will be helpful in identification and management of unrecognized kidney disease in stroke therapy.

\section{METHODOLOGY}

The present cross sectional study was carried out in the Department of Physiology, DMCH, Dhaka from July 2010 to June 2011. In this study, a total number of 200 subjects were included among them 100 apparently healthy subjects were selected as control(Group A) and 100 diagnosed acute stroke patients(both ischemic and haemorrhagic type) were selected as study group(Group B). On the basis of age group $A$ and group $B$ were further subdivided into group $A_{1}$ (age 35-59years) consisting of 47 normal persons and group $A_{2}$ (age 60-85years) consisting of 53 normal persons; group $B_{1}$ (age $35-59$ years)was consisting of 42 stroke patients and group $\mathrm{B}_{2}$ (age60-85 years) consisting of 58 stroke patients. All the stroke patients were selected from the Medicine department of $\mathrm{DMCH}$. Patients with the history of kidney disease, coma, convulsion or any other chronic illness were excluded from this study.

The purpose and procedure of the study were explained to each subject. Written informed consent was taken from all participants. Study protocol was approved by Ethical review committee of Dhaka Medical College, Dhaka. Detailed medical and family history was recorded in a prefixed questionnaire. Under aseptic precaution $5 \mathrm{ml}$ of venous blood was collected and serum was prepared for estimation of serum creatinine. The serum creatinine was measured (for measurement of eGFR) by Dimention $\AA$ clinical chemistry system at the department of physiology Dhaka Medical College, Dhaka. The eGFR was calculated by MDRD study equation [eGFR $\left(\mathrm{ml} / \mathrm{min} / 1.73 \mathrm{~m}^{2}=186 x\right.$ serum creatinine $(\mathrm{mg} / \mathrm{dl})^{-1.154} \mathrm{xage}^{-0.203} \times 0.742$ (if female) $\mathrm{x} 1.212 \times 1.212$ (if black)

Statistical analysis of data was done by SPSS program version 12. All the data were expressed as Mean \pm SD (Standard deviation) of study groups and control groups. Comparison between two groups were done by using unpaired student ' $t$ ' test . The test of significance was calculated and $p$ values $<0.05$ was accepted as level of significance.

\section{RESULTS}

The mean eGFR of group $A_{2}$ was lower than that of group $A_{1}$ and the difference was statistically significant.The mean eGFR in group $B_{1} \& B_{2}$ were lower than that of group $A_{1}$ and the differences were statistically highly significant $(p<0.001)$. The mean eGFR in group $B_{1} \& B_{2}$ were higher than that of group $A_{2}$ and the differences were statistically highly significant $(p<0.001)$. Within the study group the eGFR in group $B_{2}$ was lower than that of group $B_{1}$ but the difference was statistically significant. The mean eGFR level of group $B 2$ was statistically highly significant $(p<0.001)$ than group $A_{1}$ and $A_{2}$. (Table I).

Table I

Mean ( $\pm S D)$ eGFR $\left(\mathrm{ml} / \mathrm{min} / 1.73 \mathrm{~m}^{2}\right)$ in different groups $(n=200)$

\begin{tabular}{cll|}
\hline Groups & $\mathbf{n}$ & Mean \pm SD \\
\hline & & \\
$A_{1}$ & 47 & $107.82 \pm 20.55$ \\
$A_{2}$ & 53 & $97.47 \pm 9.62$ \\
$B_{1}$ & 42 & $61.43 \pm 29.91$ \\
$B_{2}$ & 58 & $45.96 \pm 16.26$ \\
\hline
\end{tabular}


Statistical analysis

\begin{tabular}{llrl|}
\hline Groups & $\mathbf{t}$ & df & p value \\
\hline$A_{1}$ vs $A_{2}$ & 3.286 & 98 & $0.001^{* *}$ \\
$A_{1}$ vs $B_{1}$ & 8.603 & 87 & $0.0001^{* * *}$ \\
$A_{1}$ vs $B_{2}$ & 17.226 & 103 & $0.0001^{* *}$ \\
$A_{2}$ vs $B_{1}$ & 8.258 & 93 & $0.0001^{* * *}$ \\
$A_{2}$ vs $B_{2}$ & 20.073 & 109 & $0.0001^{* * *}$ \\
$B_{1}$ vs $B_{2}$ & 3.323 & 98 & $0.001^{* *}$ \\
\hline
\end{tabular}

The results are expressed as Mean \pm SD. Unpaired Student's ' $t$ ' test was performed to compare between groups. The test of significance was calculated and $p$ values $<0.05$ was accepted as level of significance.

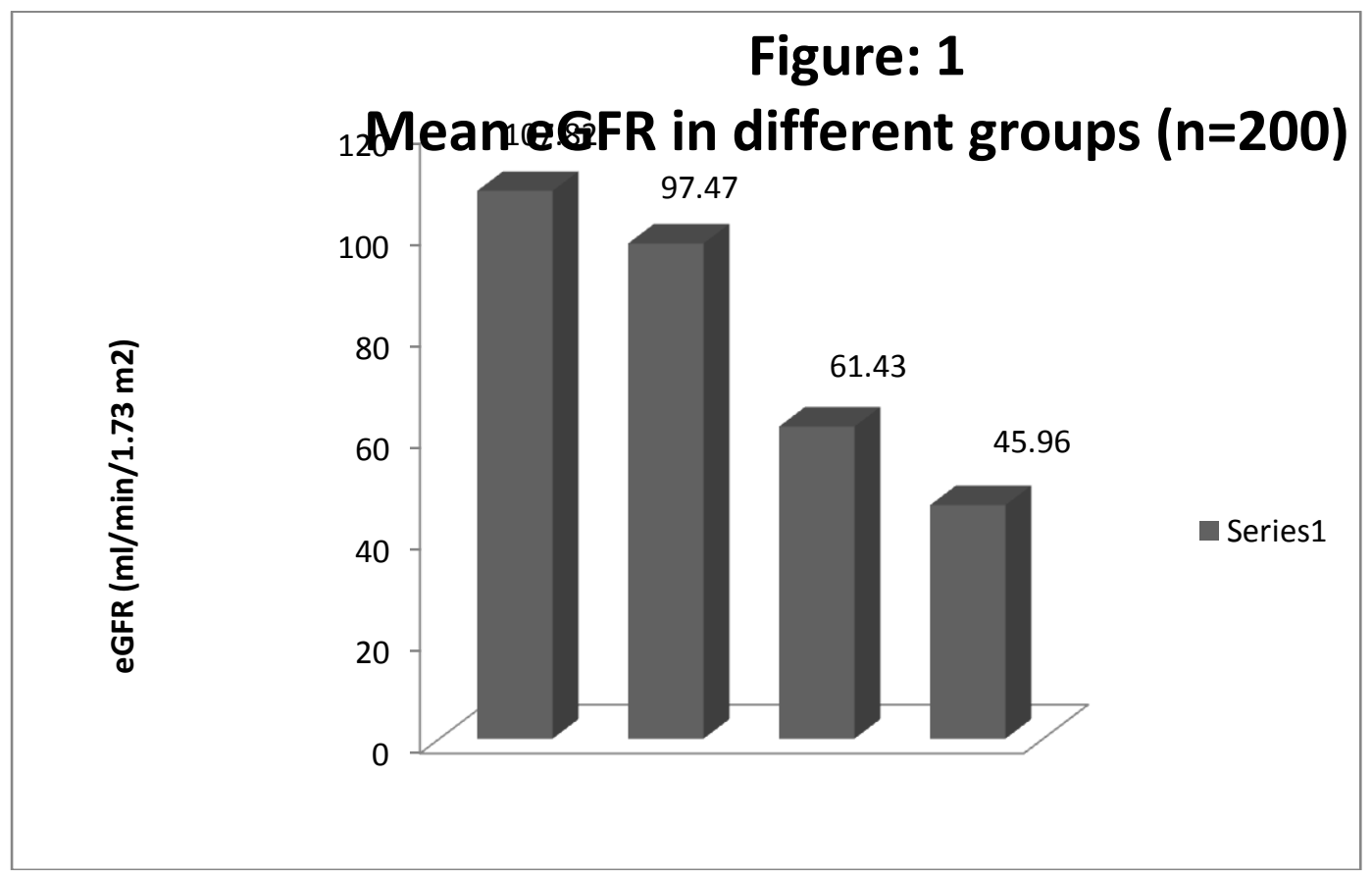

Renal function was assessed according to the Kidney disease outcomes Quality Initiative (K/DOQI)-CKD classification in five groups (US National kidney foundation guideline 2002):

Stage I Corresponded of patients with GFR Values $\geq 90 \mathrm{ml} / \mathrm{min} / 1.73 \mathrm{~m}^{2}$.

Stage II Kidney damage with mildly reduced GFR $\left(60-89 \mathrm{ml} / \mathrm{min} / 1.73 \mathrm{~m}^{2}\right)$.

StageIII Kidney damage with moderately reduced GFR $\left(30-59 \mathrm{ml} / \mathrm{min} / 1.73 \mathrm{~m}^{2}\right)$.

Stage IV Kidney damage with severely reduced GFR $\left(15-29 \mathrm{ml} / \mathrm{min} / 1.73 \mathrm{~m}^{2}\right)$.

Stage $V \quad$ Kidney failure $\left(\right.$ GFR $\left.<15 \mathrm{ml} / \mathrm{min} / 1.73 \mathrm{~m}^{2}\right)$.

In group $A_{1}, 47$ persons showed eGFR $\geq 90 \mathrm{ml} / \mathrm{min} / 1.73 \mathrm{~m}^{2}$. So, $100 \%$ people of group $A_{1}$ had normal kidney function. In group $A_{2,1,51}$ persons showed eGFR $\geq 90 \mathrm{ml} / \mathrm{min} / \mathrm{m}^{2}$ and 2 patients showed eGFR between 60-89 $\mathrm{ml} / \mathrm{min} / \mathrm{m}^{2}$.Therefore,In group $\mathrm{A}_{2} 96.2 \%$ showed normal kidney function and $3.8 \%$ people showed mildly decreased kidney function. In group $B_{1}, 7$ patients $(16.7 \%)$ had normal kidney function, 13 patients(31\%) had mildly decreased kidney function(Stage II), 20 patients(47.1\%) had moderately decreased kidney function (Stage III) and 2 patients (4.8\%)had renal insufficiency (Stage IV).In group $B_{2}, 13$ patients(22.4\%) had mildly decreased kidney function(Stage II), 37 patients(63.8\%) had moderately decreased kidney function (Stage III), and 8 patients(13.8\%) had renal insufficiency (StageIV). 
Table - II

Distribution of eGFR in different groups $(n=200)$

\begin{tabular}{c|cccc}
\hline $\begin{array}{c}\text { eGFR } \\
(\mathbf{m l} / \mathbf{m i n} / \mathbf{1 . 7 3} \\
\left.\mathbf{m}^{2}\right)\end{array}$ & $\begin{array}{c}\text { Group } \mathbf{A}_{\mathbf{1}} \\
(\mathbf{n = 4 7 )} \\
\text { Number (\%) }\end{array}$ & $\begin{array}{c}\text { Group } \mathbf{A}_{\mathbf{2}} \\
(\mathbf{n = 5 3 )} \\
\text { Number (\%) }\end{array}$ & $\begin{array}{c}\text { Group } \mathbf{B}_{\mathbf{1}} \\
(\mathbf{n = 4 2}) \\
\text { Number (\%) }\end{array}$ & $\begin{array}{c}\text { Group } \mathbf{B}_{\mathbf{2}} \\
(\mathbf{n = 5 8}) \\
\text { Number (\%) }\end{array}$ \\
\hline$\cdot 90$ & $47(100.0)$ & $51(96.2)$ & $7(16.7)$ & 0 \\
$60.00-89.99$ & 0 & $2(3.8)$ & $13(31.0)$ & $13(22.4)$ \\
$30.00-59.99$ & 0 & 0 & $20(47.6)$ & $37(63.8)$ \\
$15.00-29.99$ & 0 & 0 & $2(4.8)$ & $8(13.8)$ \\
\hline
\end{tabular}

Group $A_{1}$ : Control, age 35-60 years

$\mathrm{n} \quad=\quad$ Number of subjects

Group $A_{2}$ : $\quad$ Control, age $61-85$ years 2

Group $B_{1}$ : Case, age $35-60$ years

Group $\mathrm{B}_{2}$ : Case, age $61-85$ years

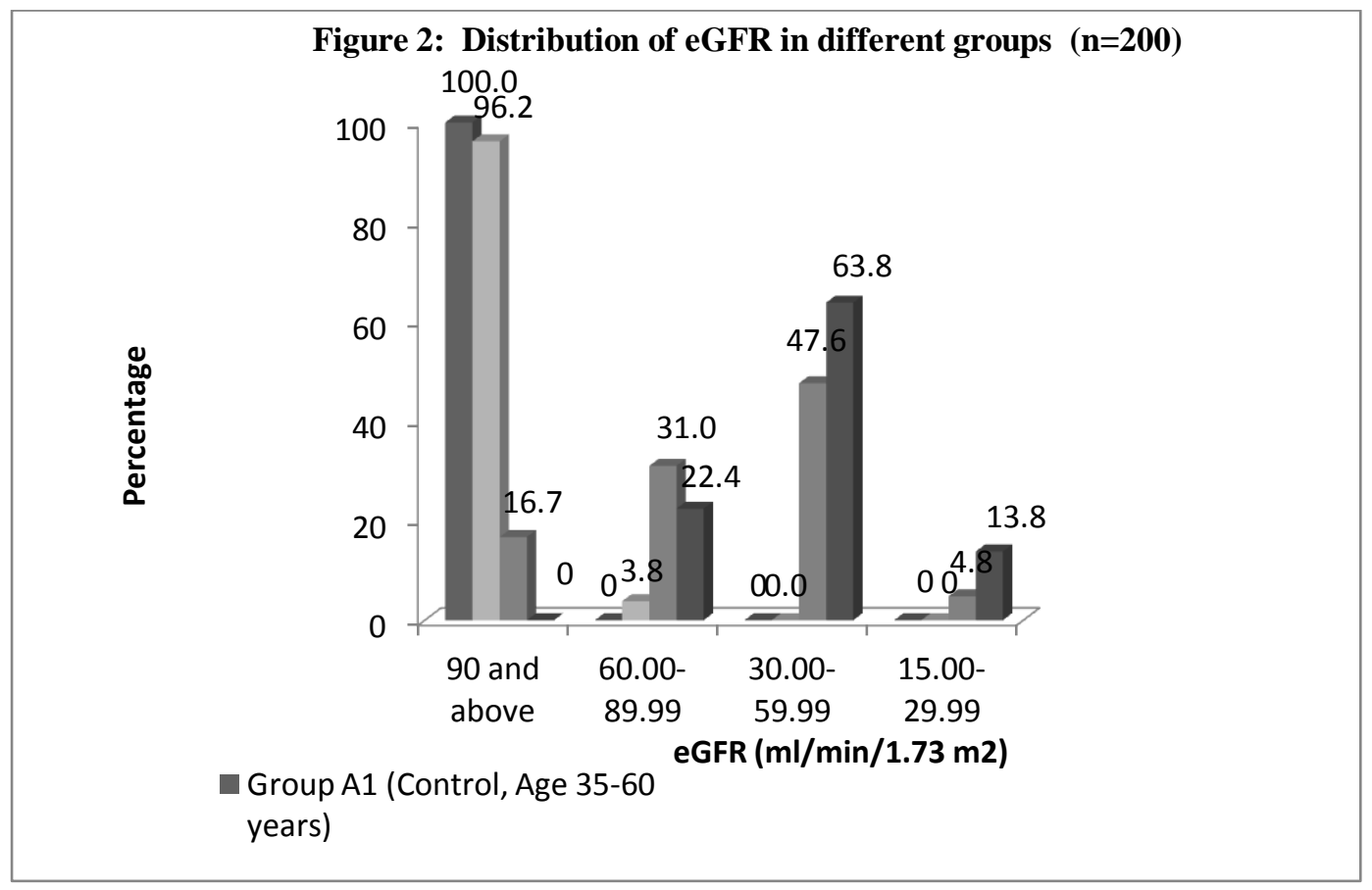

\section{DISCUSSION}

In the present study the mean eGFR of stroke patients were significantly lower $(p<0.005)$ than of the normal control group. Among the stroke patients, group $B_{2}$ patients have lower eGFR. than group $B_{1}$ patients. This findings are in consistent with other studies.

The relationship between kidney function and stroke may be shared risk factors underlying vascular disease including age, diabetes mellitus, hypertension, left ventricular hypertrophy and low density lipoprotein cholesterol. ${ }^{10}$

The vascular beds of both the kidney and brain have very low resistance and are passively perfused at high flow through systole and diastole. Because of these uniquc features which are not present in other organs, the blood vessels in the kidney and the brain are highly susceptible to flactuation in blood pressure and flow. ${ }^{11}$

Due to hemodynamic similarities between vascular beds of the kidney and the brain, small vessel disease in the kidney may be indicative of presence of small vessel disease in the brain. ${ }^{12}$ Impaired kidney function measured by decreased GFR is related to subclinical markers of cerebral small vessel disease.

Decreased GFR is related to white matter lesion, subcortical atrophy and to a lesser extent lacunar infracts Based on MRI results, Ikram et al found that, impaired kidney function is associated with MRI markers of cerebral small vessel disease and suggested that this relationship may be due to the fact that the blood vessels of both kidney 
and the brain have low resistance and are therefore highly susceptible to fluctuation of blood pressure and flow. ${ }^{13}$

Presence of CKD (Chronic kidney disease) in various forms and even at mild stages is associated with cerebrovascular disease. ${ }^{14}$

Cerebral small vessel disease is connected to kidney function as estimated by eGFR in patients hospitalized for acute stroke and small vessel disease and kidney function are closely associated with poor post stroke survival. ${ }^{15}$

Immediate period following a stroke, acute kidney injury may develop as a possible complication and confirms the importance of baseline renal function as a predictor of mortality for stroke patients. ${ }^{16}$

Lower eGFR was positively associated with increased white matter hyperintensity volume and kidney disease is an important, independent risk factor for cerebrovascular disease. ${ }^{5}$

There is high prevalence of chronic kidney disease In Chinese patients with incident cerebrovascular lesion (CVL). $47.7 \%$ of patients with CVL had eGFR<60ml/min/ $1.73 \mathrm{~m}^{2} .17$

The presence of kidney disease constitute strong independent predictors of mortality and poor outcome in patients with acute stroke. ${ }^{8}$

After acute stroke, patients with reduced eGFR had a higher mortality risk and therefore can be used to stratify risk for stroke patients. ${ }^{18}$

Impaired kidney function, measured by decreased eGFR, is related to subclinical markers of cerebral small vessel disease and eGFR might be used as an easily measurable indicator of cerebral small vessel disease. ${ }^{4}$

Among the patients with acute ischemic stroke reduced eGFR at hospital admission is associated with a higher mortality rate compared to patients with moderate levelsof eGFR. ${ }^{19}$

Reduced estimated GFR appears to be a significant independent risk factor for short as well as long term mortality. ${ }^{20}$

Therefore, eGFR could be added to the other known prognostic factors and it should be applied for identification and management of unrecognized kidney diseases in stroke therapy.

\section{CONCLUSION}

From this study, it can be concluded that, kidney dysfunction is more in stroke patients of advanced age group on the basis of measuring decreased eGFR.

\section{REFERENCES}

1. Mc Clellan W, Warnock DG, Mc Clure L. Racial Differences in the prevalence of Chronic Kidney disease among participants in the reasons for geographic and racial differences in Stroke. J Intern Med. 2001; 97(5): 293-296.
2. Oksala NK, Salonen T, Standberg T, Oksala A. Cerebral small vessel Disease predict long term survival in stroke patients. Stroke; 2010;41(9):1914-1920.

3. Buchman A, Tanne D, Boyle PA. Kidney function is associated with the rate of cognitive decline in the elderly. Neurology 2009;73:920-927.

4. Ikram MA, Vernooji MW, Hofman A. Kidney function is related to cerebral small vessel disease. Stroke 2008; 39(1): 55-61.

5. Minesh K, Clinton BW, Thomas LN. Chronic kidney diseases is associated with white matter hyperintensity volume. Stroke 2007;38(5): 3121-3126.

6. Mayumi $K$, Nobuhito $H$, Satoshi M. Silent brain infarction and rapid decline of kidney function in patients with CKD, Americal journal of kidney disease. 2010;56(3): 468-476.

7. Rule $A D$, Larson $T S$, Jacobsen $S$ J. Using serum creatinine to estimate glomerular filtration accuracy in good health and in chronic kidney disease. An intern Med.2004; 141(7):929-937.

8. Gilad Y, Roseline S, Maya T, David O. Chronic kidney disease and clinical outcome in patients with acute stroke. Stroke 2009; 40(5) 1296-1303.

9. Yalahom G, Merzeliak O, Toashi M. Low glomerular filtration rate and risk of stroke.2010; 314(45): 42-49.

10. Patrizia DF, Jean $\mathrm{CL}$, Emmanuel C. Anaemia and chronic kidney diseases are potential risk facrors for mortality in stroke patient. BMC Nephrol 2010; 11(27):68-96.

11. Ikram MA, Vernooji MW, Hofman A, Nelson WJ. Kidney function is related to cereberal small vessel disease. Stroke. 2008; 39(1): 55-61.

12. Ruyun J, Jeremiah RB, Anthony PF. Estimation of glomerular filtration rate and renal function. The Annals of Thoracic Surgery. 2008;86(1):1-3.

13. Hao Z, Wu B,Tao WD, Lin S, Kong FY. Association between renal function and clinical outcome in patient with acute stroke. Eur Neurol 2010; 63(4):237-242.

14. Meng L, Bruce $O$, Reno-cerebrovascular disease: linking the nephron with neuron. Summary expert review of neurotherapeutics.2011; 11(2):241-249.

15. Niku KJ, Tapani S, Timo S, Anni O. Cerebral small vessel disease predicts long term survival in stroke patient. American Heart Association.2010; 41(2):97-115.

16. Adrian $\mathrm{C}$, Adalbert $\mathrm{S}$, Nicolete GM,Ligia P.The impact of acute kidney injury on short term survival in an eastern European population with stroke. Nephrol Dial Transplant. 2007; 23(5) 2228-2234.

17. Jing $X$, Weiming W, Hao S, Shengdi C.Chronic kidney disease is prevalent in Chinese patients admitted with verified cerebrovascular lesions and predicts short term prognosis. Ann Neurol. 1991;30(5) 725-730.

18. Ronald S, Mac W, Suzzane YS,Graham S. Does renal dysfunction predict mortality after acute stroke? Stroke 2002; 33(3): 16301635.

19. Mostfsky E, Welenius GA,Noheria A, Levitan EB. Renal function predicts survival in patients with acute stroke. Cerebrovascular Dis. 2009;28(1):88-94.

20. George T, Theodore A, Maris A, Kimon S. Renal dysfunction in acute stroke: an independent predictor of long term all combined vascular events and overall mortality. Nephrol Dial Transplant $2009 ; 24(8) 194-200$ 